\title{
Time evolution of a medium-modified jet
}

\section{Liliana Apolinário* \\ LIP}

Av. Prof. Gama Pinto, n.2 Complexo Interdisciplinar(3is) 1649-003 Lisboa Portugal E-mail:

liliana@lip.pt

\begin{abstract}
The presence of a hot and dense medium, produced in ultra-relativistic heavy-ion collisions, is known to modify the parton shower evolution. Several observations of the resulting intra-jet activity show significant modifications of what can be considered as a medium-modified jet from a "vacuum" (proton-proton) reference. These modifications, generically known as jet quenching effects, are the result of the multiple interactions of the parton shower with the produced fastevolving quark-gluon plasma (QGP). Recent efforts have tried to assess the time dependence of jet quenching effects, with particular focus on late or early dynamics. In this talk, we show a novel tool that can potentially evaluate the full-time evolution of the QGP by applying jet reclustering techniques. The result can bring novel insights into the QGP expansion as well as shed some light on how to re-sum, in a consistent way, vacuum-like and medium-like emissions into a single parton shower evolution equation.
\end{abstract}

European Physical Society Conference on High Energy Physics - EPS-HEP2019 -

10-17 July, 2019

Ghent, Belgium

${ }^{*}$ Speaker. 


\section{Introduction}

In ultrarelativistic heavy-ion collisions, extreme temperatures and densities allow the production of a hot and dense liquid of quarks and gluons, called the Quark-Gluon Plasma (QGP). With an initial volume proportional to the overlap of the incoming nuclei, this state of matter quickly expands, dropping its temperature bellow the deconfinement transition (or cross-over), forming a high multiplicity event. Due to its short lifetime, its study is made only indirectly by analyzing the final particles that are the result of the QGP production and expansion (generically known as soft probes). Alternatively, one can use high momentum particles that are produced simultaneously within the collision (hard probes). By evaluating their modifications concerning a reference (usually, proton-proton collisions), it is possible to infer the properties of the medium that these probes traveled through.

The Relativistic Heavy-Ion Collider (RHIC) and the Large Hadron Collider (LHC), collected a paramount of results in both sectors. These include hydrodynamic flow patterns, suppression of heavy-quark bound states, hadrochemistry of the final state, and modifications of the fragmentation of energetic partons that traverse the medium (see [1,2] for an overview of the results). Together, they brought unique insight into the properties of the QGP (see $[3,4,5,6])$. However, a limiting factor of all these observables is that they are the integrated result of a fast-evolving and extended medium. As such, there is a strong time-dependence of the medium properties that require novel strategies to finally measure the time-structure of the QGP.

\section{Probing the QGP time evolution}

Attempts to probe the space-time evolution of the QGP have been put forward recently. In particular, the works by [7] and [8].

In the former, using a framework to change quenching during early stages (based on quenching weights), the authors show that the nuclear modification factor, $R_{A A}$ seems to be insensitive to a "switching off" of quenching effects during the first $0.6 \mathrm{fm} / \mathrm{c}$. Conversely, the high momentum anisotropic flow appears to be quite sensitive to such change. In particular, the scenario in which the initialization time for energy loss effects starts after $0.6 \mathrm{fm} / \mathrm{c}$ seems to be preferred by both ATLAS and CMS results. This observation shows the potential to constrain the dynamics of the initial stages of medium evolution.

In the later, there is a proposal to use semi-leptonic decay of $t \bar{t}$ events as a tomographic probe of the QGP. In particular, this constitutes a time-delayed probe of the medium as it is feasible to constrain the time at which the (colorless) $W$ boson decays into a $q \bar{q}$ pair. By selecting higher momentum particle (and so highly boosted particles), one can trigger on reconstructed $W$ bosons that are produced at later times. As such, its hadronic products will interact with the resulting QGP that is, by then, already in a quite diluted state. The result will be a shifted reconstructed $W$ mass that will depend on the $w$ decay point. The result will evolve between a framework in which particles start to interact with the medium immediately after its production (fully quenching scenario) and vacuum expectations (proton-proton). If the former is known (from, for instance, boson-jet measurements), By measuring the reconstructed W-boson mass as a function of the top transverse momentum, it should be possible to perform a full tomographic analysis of the QGP 
time evolution. While this result shows that in the Future Circular Collider, a scan of the entire QGP lifetime is possible, currently, even with the HE-LHC upgrade, there is limited discrimination between short and long-lived mediums.

As such, nowadays, there are possible exploration avenues to constrain initial and later times of the QGP evolution. But it is desirable (and highly needed) to have a probing tool that would allow slicing different QGP timescales more directly. In the next section, we will try to argue that among our current probes, jets might be a good candidate for such studies.

\section{Jets: a multi-scale probe}

Jet production is quite abundant at both RHIC and the LHC. They are a space-time evolving structure that is the result of a QCD parton shower. As such, a jet can be considered as a number of multiple parton emissions, until a lower energy scale (when non-perturbative processes become relevant).

In a perturbative formulation, such iterative process is, at leading order, governed by angular ordered emissions, such that:

$$
d N^{\omega \rightarrow 0} \sim \alpha_{S} C_{F} \frac{d \omega}{\omega} \frac{\sin \theta d \theta}{1-\cos \theta} \Theta\left(\cos \theta-\cos \theta_{q \bar{q}}\right)
$$

describes the spectrum of radiated soft gluons, with energy $\omega$ and angle $\theta$, off a quark that was previously emitted with angle $\theta_{q \bar{q}}$.

The time for a given emission to be regarded as an independent source of emission can be estimated as the lifetime of the virtual quark-gluon state. In the center-of-mass reference frame of the virtual pair, this time goes as:

$$
\Delta t \sim \frac{1}{\Delta E} \sim \frac{1}{m_{\text {virtual }}} .
$$

In the laboratory frame, due to the Lorentz boost, this parametric estimate yields:

$$
\tau_{\text {form }} \simeq \frac{1}{z(1-z) E \theta^{2}},
$$

where $E$ is the energy of the parent quark, $z$ the energy fraction carried by the gluon and $\theta$ the angle between the final quark and gluon. As such, the full parton shower should encode all such parametric estimates for lifetimes in a somehow ordered way (at leading order).

\subsection{Vacuum vs in-medium parton shower}

In vacuum, the Monte Carlo approach of iterative parton emissions does not have a physical realization. The probabilistic interpretation of quantum emissions, driven by an ordering variable (virtuality, transverse momentum, or angle), is known to capture the final picture of a jet, i.e, the set of final particles. Because the whole transformation process of a single parton to a collection of final state particles takes place without an intermediate measure of the process, it is not known if the iterative process of the Monte Carlo approach had that particular realization. However, if the same process now takes place inside a medium whose density is evolving with time, it might be possible to relate the characteristics of a given parton emission with the medium density that 
participated in the emission of that given particle. From there, one should be able to capture the concept of a physical time in the parton shower development.

As a first study along this line, we used a vacuum Monte Carlo event generator (PYTHIA8.2.40 [9]) and a Monte Carlo event generator that includes medium-induced effects to simulate how the jet is modified by the presence of a QGP (JEWEL 2.2.0 [10]). As a first study, and as a proof of concept, we choose not to include medium-recoil effects to facilitate the tracking/identification of particles that come exclusively from the parton shower. Future studies will naturally include these effects.

For the results presented in this manuscript, it was simulated di-jets events at $5.02 \mathrm{TeV}$ at hadron level ([0-10]\% centrality class within JEWEL and pre-defined simple medium parameters). The list of final jets is obtained using the anti- $k_{t}$ algorithm[11], with radius $R=0.5$, a minimum transverse momentum of $p_{T, \min }=100 \mathrm{GeV}$ and a pseudo-rapidity window of $|\eta|<1$, performed within the FastJet package[12]. The choice of such a narrow window is to reduce effects that are not Lorentz invariant. In order to have already a time structure within the jet, we decided to recluster the jets with the generalized $k_{t}$ jet algorithm:

$$
d_{i j}=\min \left(p_{t, i}^{2 p}, p_{t, j}^{2 p}\right) \frac{\Delta R_{i j}^{2}}{R^{2}}, d_{i, B}=p_{t, i}^{2 p},
$$

with $p=0.2$. This value provides the same parametric estimate that the inverse of $\tau_{\text {form }}$ :

$$
d_{i j} \sim p_{T} \theta^{2} \sim \tau_{\text {form }}^{-1}
$$

While this choice might appear as unorthodox for a vacuum parton shower, we are already anticipating the physics of a medium-modified parton shower. JEWEL already includes some formation time effects for subsequent emissions. In particular, the interplay between competing radiative processes is governed by the formation time (the emission with a longer formation time is discarded). For the vacuum parton shower, since $\tau_{\text {form }}$, as it is defined, depends intrinsically on the kinematical properties of the radiated quanta (such as emission angle and transverse momentum), we expect that this reclustering also captures QCD-inspired splittings, somehow ordered from long to short $\tau_{\text {form }}$. Naturally, other reclustering algorithms (such as Cambridge-Aachen[13, 14]), might also be investigated.

\subsection{Jet Splitting function}

With the procedure from the last section, and selecting only the leading jet, we recursively identify the sub-jet prongs from the primary branch by unclustering the jet sequence. Two different $\tau_{\text {form }}$ intervals were selected: $\tau_{\text {form }}<2.5 \mathrm{fm} / \mathrm{c}$ and $\tau_{\text {form }}>5 \mathrm{fm} / \mathrm{c}$. These values were chosen to select splittings that took place inside the medium and splittings that were, in its most, driven by vacuum physics (or in a state where the QGP is already very diluted). The corresponding fragmentation function of the sub-jets that fall into each window is shown in figure 1. In this plot, $\xi=\log (1 / z)$, with $z$ being the fraction of energy carried by the sub-leading jet. The solid corresponds to the interval of small $\tau_{\text {form }}$ while the dashed to the splittings with a formation time that falls into the window of $\tau_{\text {form }}>5 \mathrm{fm} / \mathrm{c}$. While the later does now show any significant modification between JEWEL (in red, labeled medium) and PYTHIA (in blue, labeled vacuum), we see a clear 


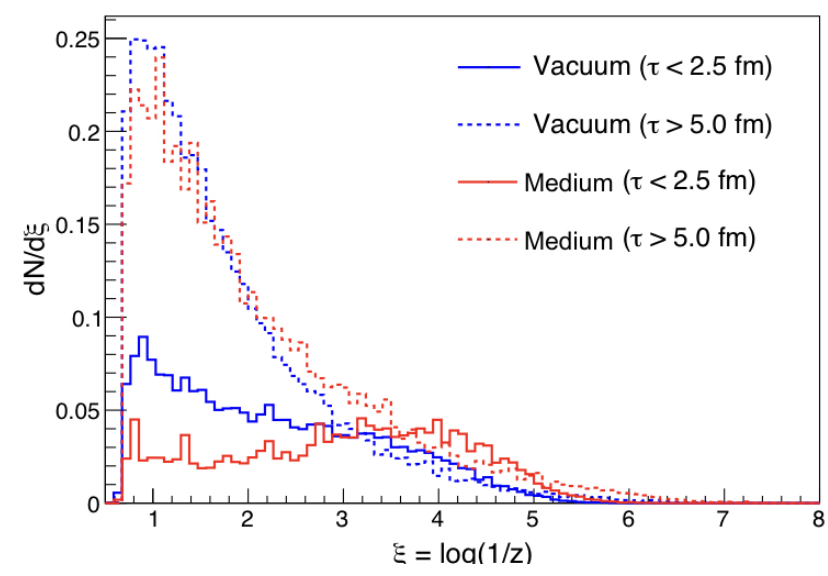

Figure 1: Fragmentation function of the jet recursive splittings obtained from the primary branch when using a generalised $k_{t}$ algorithm with $p=1$. The $z$ is the energy fraction of the sub-leading sub-jet. The label Vacuum corresponds to PYTHIA8 and Medium to JEWEL2.2.0. Two intervals of formation time are also shown: $\tau_{\text {form }}=\tau<2.5 \mathrm{fm} / \mathrm{c}$ and $\tau_{\text {form }}=\tau>5.0 \mathrm{fm} / \mathrm{c}$.

modification of this distribution when we move to sub-jets that were formed early, within the QGP lifetime.

As a simple exercise to verify that the characteristics of the radiation could be potentially related to the time evolution of the QGP, we took the average value of the above distribution, $\langle\xi\rangle$, and plotted the ratio medium over vacuum as a function of the obtained formation time. This is shown in figure 2, for 3 intervals of $\tau_{\text {form }}:[0-2.5] \mathrm{fm} / \mathrm{c} ;[2.5-5] \mathrm{fm} / \mathrm{c} ;[5-9] \mathrm{fm} / \mathrm{c}$. There is clear linear depletion that is approaching to 1 , as expected, suggesting the dilution of the QGP density that is incorporated within JEWEL (a Bjorken expansion). Naturally, the units in $x$-axis are to be taken as parametric estimates. Moreover, the $\tau_{f o r m}$ itself is obtained using a generalised $k_{t}$ reclustering. Being so, the kinematic information that is retrieved from each of the sub-jets in the process of successive unclustering is also affected by this choice.

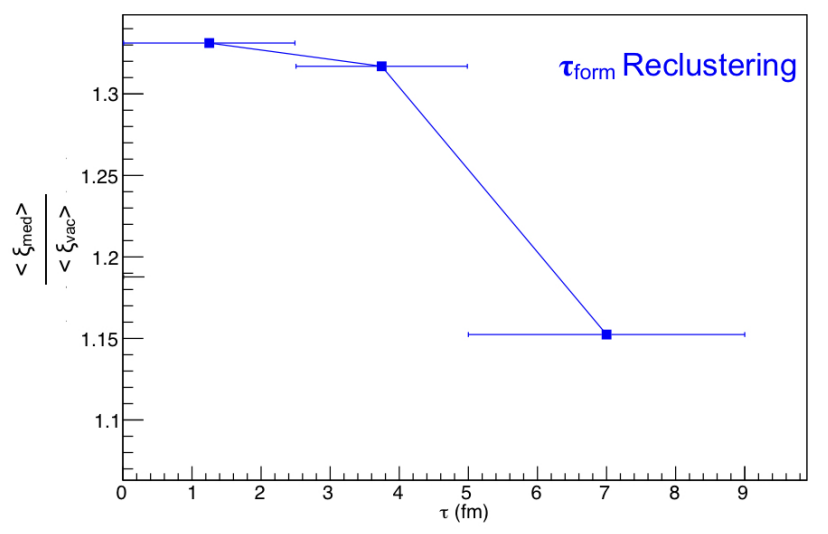

Figure 2: Medium-to-Vacuum ratio of the average value of $\xi$ (to be read from figure 1), as a function of formation time. 
With this concern in mind, it was evaluated how well the reclustered sub-jets would correspond to the emissions in the Monte Carlo history. Again, caution should be taken when interpreting the obtained results as the Monte Carlo history is not meant to reproduce the exact physical process in each quantum emission, but the final result of the whole parton shower. Moreover, the iterative soft drop process is known to yield a jet splitting function $\left(z_{g}\right)[15,16]$ that is comparable to the QCD splitting functions in proton-proton collisions. While the same measurement has been reported in heavy-ions, the link between this measurement and that to a QCD-like splitting is still unclear[17]. However, one can take this exercise to understand if QCD-like emissions are tagged in the same way when using this reclustering algorithm $(p=1)$. In this part, we took only PYTHIA and the corresponding correlation plot between the formation time that is computed within the Monte Carlo event generator ( $y$-axis) and the un-clustering method ( $x$-axis) is shown in figure 3 . There is indeed a direct correlation between the two quantities, on top of some wrong branch identifications that spread along the values of $\tau_{\text {form }}$. Another key feature of this figure is a vertical line for shorter formation times when doing the un-clustering. These splittings correspond to large angle radiations that will not be part of the jet as they will end in a region of $(\eta, \phi)$ phase space at an $R>0.5$ from the jet axis.

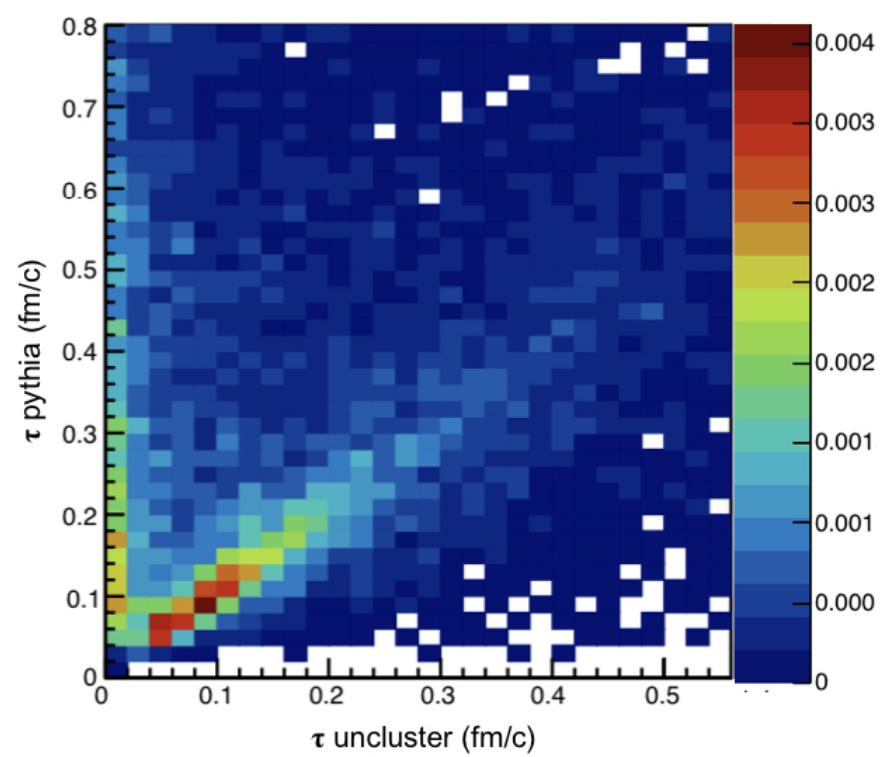

Figure 3: Correlation between the formation time obtained from the jet unclustering method ( $x$-axis) and the PYTHIA8 parton shower history ( $y$-axis).

\section{Conclusions}

One of the goals of the heavy-ion program in ultra-relativistic heavy-ion collisions is to measure the QGP properties accurately. Since the produced medium is fast expanding, aiming for precision measurements requires an evaluation of the time-dependence of the medium characteristics. Therefore, there is an urgent need to evaluate the QGP space-time evolution.

In this work, we made a first attempt to use jet substructure as a proxy for the time evolution of the QGP. The results show that selecting jet prongs with different formation times can indeed 
be used to quantify the effect of the QGP on those splittings. In particular, prongs with longer formation have a fragmentation function that is similar when comparing PYTHIA and JEWEL, while sub-jets with shorter formation times show distributions that are heavily modified. This can provide an exploration avenue to measure the time density profile of the medium. While the results seem promising, there are several challenges ahead. The matching between the parton shower branch (from the Monte Carlo event generator) and that to the reconstructed sub-jet was investigated within PYTHIA yielding moderate results ${ }^{1}$. Further investigation is required to check such universality across different quenching models. This is under investigation and the results will be released in a future manuscript.

Acknowledgements: The author would like to thank to R. Conceição, G. Milhano and J. Thaller for useful discussions. This work was supported by the Portuguese Fundação para a Ciência e Tecnologia under contracts DL57/2016/CP/CT and CERN/FIS-PAR/0022/2017.

\section{References}

[1] P. Foka and M. A. Janik, An overview of experimental results from ultra-relativistic heavy-ion collisions at the CERN LHC: Bulk properties and dynamical evolution, Rev. Phys. 1 (2016) 154 [1702.07233].

[2] P. Foka and M. A. Janik, An overview of experimental results from ultra-relativistic heavy-ion collisions at the CERN LHC: Hard probes, Rev. Phys. 1 (2016) 172 [1702 . 07231].

[3] M. Van Leeuwen, Highlights from the ALICE experiment, these proceedings .

[4] D. Perepelitsa, High-density QCD Matter, these proceedings .

[5] J. Grosse-Oetringhaus, Emergence of Quark-Gluon Plasma Phenomena, these proceedings .

[6] C. Salgado, Dense QCD Matter in Heavy Ion collisions and Neutron Stars, these proceedings .

[7] C. Andrés, N. Armesto, M. Luzum, C. A. Salgado and P. Zurita, Energy versus centrality dependence of the jet quenching parameter $\hat{q}$ at RHIC and LHC: a new puzzle?, Eur. Phys. J. C76 (2016) 475 [1606.04837].

[8] L. Apolinário, J. G. Milhano, G. P. Salam and C. A. Salgado, Probing the time structure of the quark-gluon plasma with top quarks, Phys. Rev. Lett. 120 (2018) 232301 [1711.03105].

[9] T. Sjöstrand, S. Mrenna and P. Skands, Pythia 6.4 physics and manual, Journal of High Energy Physics 2006 (2006) 026?026.

[10] K. C. Zapp, JEWEL 2.0.0: directions for use, Eur. Phys. J. C74 (2014) 2762 [1311. 0048 ].

[11] M. Cacciari, G. P. Salam and G. Soyez, The anti-ktjet clustering algorithm, Journal of High Energy Physics 2008 (2008) 063 ?063.

[12] M. Cacciari, G. P. Salam and G. Soyez, Fastjet user manual, The European Physical Journal C 72 (2012) .

[13] Y. L. Dokshitzer, G. D. Leder, S. Moretti and B. R. Webber, Better jet clustering algorithms, JHEP 08 (1997) 001 [hep-ph/9707323].

\footnotetext{
${ }^{1}$ At the time of the writing of these proceedings, there was a dedicated workshop to investigate the space-time structure of jet quenching[18]. Results accomplished during the meeting showed that jet grooming techniques could significantly improve the matching history between Monte Carlo event generators and jet reclustering.
} 
[14] M. Wobisch and T. Wengler, Hadronization corrections to jet cross-sections in deep inelastic scattering, in Monte Carlo generators for HERA physics. Proceedings, Workshop, Hamburg, Germany, 1998-1999, pp. 270-279, 1998, hep-ph/9907280.

[15] CMS collaboration, A. M. Sirunyan et al., Measurement of the Splitting Function in pp and Pb-Pb Collisions at $\sqrt{s_{\mathrm{NN}}}=5.02 \mathrm{TeV}$, Phys. Rev. Lett. 120 (2018) 142302 [1708.09429].

[16] ALICE collaboration, S. Acharya et al., Exploration of jet substructure using iterative declustering in pp and Pb-Pb collisions at LHC energies, 1905. 02512.

[17] G. Milhano, U. A. Wiedemann and K. C. Zapp, Sensitivity of jet substructure to jet-induced medium response, Phys. Lett. B779 (2018) 409 [1707. 04142].

[18] "Rapid reaction task force: The space-time structure of jet quenching - theory and experiment." https://indico.gsi.de/event/9065/. 